\title{
Permanent Genetic Resources added to Molecular Ecology Resources Database 1 August 2009-30 September 2009
}

Molecular Ecology Resources Primer Development Consortium, ${ }^{1}$ DOUKary abdoullaye, ${ }^{2}$ I. ACEVeDO ${ }^{3}$ ABISOLA A. ADEBAYO ${ }^{4}{ }^{\mathrm{JASM}} \mathrm{ANCA}$ BEHRMANN-GODEL,${ }^{5}$ R. C. BENJAMIN, ${ }^{6}$ DAN G. BOCK, ${ }^{4}$ CÉLINE BORN, ${ }^{7}$ CARINE BROUAT,${ }^{8}$ ADALGISA CACCONE, ${ }^{9}$ LING-ZHEN CAO,${ }^{10}$ P. CASADOAMEZÚA ${ }^{3}{ }^{3}$. CATANÉO,${ }^{11}$ M. M. CORREA-RAMIREZ, ${ }^{12,13}$ MELANIA E. CRISTESCU,${ }^{4}$ GAUTHIER DOBIGNY,${ }^{8,14}$ EMMANUEL E. EGBOSIMBA,${ }^{4}$ LIANNA K. ETCHBERGER, ${ }^{15}$ BIN FAN,${ }^{16}$ PETER D. FIELDS, ${ }^{17}$ D. FORCIOLI, ${ }^{11}$ P. FURLA, ${ }^{11}$ F. J. GARCIA DE LEON, ${ }^{12}$ R. GARCÍA-JIMÉNEZ, ${ }^{3}$ PHILIPPE GAUTHIER,${ }^{8}$ RENÉ GERGS,${ }^{5}$ CLEMENTINA GONZÁLEZ, ${ }^{18,19}$ LAURENT GRANJON,${ }^{2,8}$ CARLA GUTIÉRREZ-RODRÍGUEZ, ${ }^{18}$ NATHAN P. HAVILL,${ }^{20}$ P. HELSEN, ${ }^{21}$ TYLER D. HETHER, ${ }^{22}$ ERIC A. HOFFMAN, ${ }^{22}$ XIANGYANG HU, ${ }^{23}$ PÄR K. INGVARSSON,${ }^{24}$ S. ISHIZAKI, ${ }^{25}$ HEYI JI, ${ }^{26}$ X. S. JI, ${ }^{27}$ M. L. JIMENEZ, ${ }^{13}$ R. KAPIL, ${ }^{6}$ R. KARBAN, ${ }^{28}$ STEPHEN R. KELLER, ${ }^{17}$ S. KUBOTA, ${ }^{25}$ SHUZHEN LI, ${ }^{26}$ WANSHA LI, ${ }^{23}$ DOUGLAS D. LIM, ${ }^{29}$ HAORAN LIN, ${ }^{16}$ XIAOCHUN LIU, ${ }^{16}$ YAYAN LUO, ${ }^{16}$ A. MACHORDOM, ${ }^{3}$ ANDREW P. MARTIN, ${ }^{29}$ E. MATTHYSEN, ${ }^{21}$ MAXWELL N. MAZZELLA, ${ }^{29}$ MÉLODIE A. MCGEOCH,${ }^{30,31}$ ZINING MENG,${ }^{16} \mathrm{M}$. NISHIZAWA, ${ }^{25}$ PATRICIA O'BRIEN, ${ }^{32} \mathrm{M}$. OHARA, ${ }^{25}$ JUAN FRANCISCO ORNELAS, ${ }^{18} \mathrm{M}$. F. ORTU, ${ }^{11}$ AMY B. PEDERSEN,${ }^{33} \mathrm{~L}$. PRESTON, ${ }^{6}$ QIN REN,${ }^{10} \mathrm{KARL-OTTO}$ ROTHHAUPT, ${ }^{5}$ LOREN C. SACKETT, ${ }^{29}$ QING SANG,${ }^{16}$ G. M. SAWYER, ${ }^{6}$ K. SHIOJIRI, ${ }^{34}$ DOUGLAS R. TAYLOR, ${ }^{17} \mathrm{~S}$. VAN DONGEN, ${ }^{21}$ BETTINE JANSEN VAN VUUREN, ${ }^{7,31} \mathrm{~S}$. VANDEWOESTIJNE, ${ }^{35}$ H. WANG, ${ }^{27}$ J. T. WANG, ${ }^{27}$ LEWANG,${ }^{16}$ XIANG-LI XU, ${ }^{10}$ GUANG YANG, ${ }^{26}$ YONGPING YANG, ${ }^{23}$ Y. Q. ZENG ${ }^{27}$ QING-WEN ZHANG, ${ }^{10}$ YONGPING ZHANG, ${ }^{16}$ Y. ZHAO ${ }^{27}$ and YAN ZHOU, ${ }^{23}$ ${ }^{1} 6270$ University Blvd, Vancouver, BC, V6T 1Z4, Canada; ${ }^{2}$ I.R.D., Centre de Biologie et de Gestion des Populations, BP 2528, Bamako, Mali; ${ }^{3}$ Departamento de Biodiversidad y Biología Evolutiva, Museo Nacional de Ciencias Naturales (CSIC), José Gutiérrez Abascal, 2, 28006, Madrid, Spain; ${ }^{4}$ University of Windsor - Great Lakes Institute for Environmental Research, Windsor, ON, N9B 3P4, Canada; ${ }^{5}$ Limnological Institute, University of Konstanz, D-78467 Konstanz, Germany; ${ }^{6}$ Department of Biological Sciences, University of North Texas, 1155 Union Circle, \#305220, Denton, TX 76203-5017, USA; ${ }^{7}$ Evolutionary Genomics Group, Department of Botany and Zoology, Stellenbosch University, Private Bag, X1, Matieland 7602, South Africa; ${ }^{8}$ Institut de Recherche pour le Développement (I.R.D.), Centre de Biologie et de Gestion des Populations (UMR IRD/INRA/Cirad/SupAgro), Campus International de Baillarguet CS30016, 34988 Montferrier-sur-Lez, France; ${ }^{9}$ Department of Ecology and Evolutionary Biology, Yale University, New Haven, CT 06520, USA; ${ }^{10}$ College of Agronomy and Biotechnology, China Agricultural University, 100193, Beijing, China; ${ }^{11}$ E.A. 4228 ECOMERS, Université de Nice-Sophia Antipolis, Faculté de Sciences, Parc Valrose, BP 71, F-06108 Nice Cedex, France; ${ }^{12}$ Laboratorio de Genetica de la Conservacion, Centro de Investigaciones Biologicas del Noroeste Mar Bermejo 195, Col. Playa Palo de Santa Rita; La Paz, B.C.S. 23090, Mexico; ${ }^{13}$ Laboratorio de Aracnologia y Entomologia, Centro de Investigaciones Biologicas del Noroeste Mar Bermejo 195, Col. Playa Palo de Santa Rita; La Paz, B.C.S. 23090, Mexico; ${ }^{14}$ I.R.D., Centre Régional Agrhymet, Rive droite, BP11011, Niamey, Niger, ${ }^{15}$ Department of Biology, Utah State University, Uintah Basin Regional Campus, 1680 West Highway 40, Vernal, UT 84078, USA; ${ }^{16}$ State Key Laboratory of Biocontrol, Institute of Aquatic Economic Animals and the Guangdong Province Key Laboratory for Aquatic Economic Animals, School of Life Sciences, Sun Yat-Sen University, Guangzhou, China; ${ }^{17}$ Department of Biology, University of Virginia, Charlottesville, VA 22904, USA; ${ }^{18}$ Departamento de Biología Evolutiva, Instituto de Ecología, A.C. Km 2.5 Carretera Antigua a Coatepec No. 351, Congregación El Haya, Xalapa, Veracruz 91070, México; ${ }^{19}$ Doctorado en Ciencias Biomédicas, Universidad Nacional Autónoma de México (UNAM), México, D. F. 04510, México; ${ }^{20}$ USDA Forest Service Northern Research Station, Hamden, CT 06514, USA; ${ }^{21}$ University of Antwerp, Evolutionary Ecology Group, Groenenborgerlaan 171, 2020 Antwerp, Belgium; ${ }^{22}$ Department of Biology, University of Central Florida, 4000 Central Florida Blvd., Orlando, FL 32816, USA; ${ }^{23}$ Kunming Institute of Botany, Institute of Tibet Plateau Research at Kunming, Chinese Academy, Kunming, China; ${ }^{24}$ Department of Ecology and Environmental Science, Umeå Plant Science Centre, Umeå University, SE-901 87 Umeå, Sweden; ${ }^{25}$ Graduate School of Environmental Science, Hokkaido University, Sapporo, Hokkaido, 060-0810, Japan; ${ }^{26}$ Jiangsu Key Laboratory for Biodiversity and Biotechnology, College of Life Sciences, Nanjing Normal University, Nanjing 210046, China; ${ }^{27}$ College of Animal Science and Technology, Shandong Agricultural University, Taian 271018, China; ${ }^{28}$ Department of Entomology, University of California, Davis, CA 95616, USA; ${ }^{29}$ Department of Ecology and Evolutionary Biology, University of Colorado, N122 Ramaley, UCB 334, Boulder, CO 80309, USA; ${ }^{30}$ Cape Research Centre, South 
African National Parks, P.O. Box 216, Steenberg 7947, South Africa; ${ }^{31}$ Department of Botany and Zoology, Centre for Invasion Biology, Stellenbosch University, Private Bag X1, Matieland 7602, South Africa; ${ }^{32}$ Center of Veterinary Science, FISH Lab, Madingley Road, Cambridge CB3 OES, UK; ${ }^{33}$ Centre for Infection, Immunity and Evolution, Institute of Evolutionary Biology, University of Edinburgh, Ashworth Laboratories, Kings Buildings, Edinburgh EH10 5HN, UK; ${ }^{34}$ Center of Ecological Research, Kyoto University, Otsu 520-2113, Japan; ${ }^{35}$ Université catholique de Louvain, Biodiversity Research Centre, Place Croix du Sud 4-5, 1348 Louvain-la-Neuve, Belgium

\begin{abstract}
This article documents the addition of 238 microsatellite marker loci and 72 pairs of Single Nucleotide Polymorphism (SNP) sequencing primers to the Molecular Ecology Resources Database. Loci were developed for the following species: Adelges tsugae, Artemisia tridentata, Astroides calycularis, Azorella selago, Botryllus schlosseri, Botrylloides violaceus, Cardiocrinum cordatum var. glehnii, Campylopterus curvipennis, Colocasia esculenta, Cynomys ludovicianus, Cynomys leucurus, Cynomys gunnisoni, Epinephelus coioides, Eunicella singularis, Gammarus pulex, Homoeosoma nebulella, Hyla squirella, Lateolabrax japonicus, Mastomys erythroleucus, Pararge aegeria, Pardosa sierra, Phoenicopterus ruber ruber and Silene latifolia. These loci were cross-tested on the following species: Adelges abietis, Adelges cooleyi, Adelges piceae, Pineus pini, Pineus strobi, Tubastrea micrantha, three other Tubastrea species, Botrylloides fuscus, Botrylloides simodensis, Campylopterus hemileucurus, Campylopterus rufus, Campylopterus largipennis, Campylopterus villaviscensio, Phaethornis longuemareus, Florisuga mellivora, Lampornis amethystinus, Amazilia cyanocephala, Archilochus colubris, Epinephelus lanceolatus, Epinephelus fuscoguttatus, Symbiodinium temperate-A clade, Gammarus fossarum, Gammarus roeselii, Dikerogammarus villosus and Limnomysis benedeni. This article also documents the addition of 72 sequencing primer pairs and 52 allele specific primers for Neophocaena phocaenoides.
\end{abstract}

This article documents the addition of 238 microsatellite marker loci and 72 pairs of Single Nucleotide Polymorphism (SNP) genotyping primers to the Molecular Ecology Resources Database. Table 1 contains information on the focal species, the number of loci developed, any other species the loci were tested in and the accession numbers for the loci in both the Molecular Ecology Resources Database and GenBank. The authors responsible for each set of loci are listed in the final column. Table 2 presents information on
SNP genotyping resources added to the MER database, and presents data on the focal species, the number of sequencing primer pairs, the observed number of SNPs, other species the loci were tested in, and the number of allele specific primers or probes. The MER database and Genbank accession numbers and the authors responsible are also listed. A full description of the development protocol for the loci presented here can be found on the Molecular Ecology Resources Database (http://tomato.biol.trinity.edu/). 
Table 1 Information on the focal species, the number of loci developed, any other species the loci were tested in and the accession numbers for the loci in both the Molecular Ecology Resources Database and GenBank. The authors responsible for each set of loci are listed in the final column

\begin{tabular}{|c|c|c|c|c|c|}
\hline Species & $\begin{array}{l}\text { No. of } \\
\text { primers } \\
\text { developed }\end{array}$ & $\begin{array}{l}\text { Other } \\
\text { species } \\
\text { tested }\end{array}$ & $\begin{array}{l}\text { MER } \\
\text { database } \\
\text { no. }\end{array}$ & $\begin{array}{l}\text { GenBank } \\
\text { Accession } \\
\text { no. }\end{array}$ & Authors \\
\hline Adelges tsugae & 16 & $\begin{array}{l}\text { A. abietis, } \\
\text { A. cooleyi, } \\
\text { A. piceae, } \\
\text { Pineus pini, } \\
\text { P. strobi }\end{array}$ & 37980-37995 & $\begin{array}{l}\text { GQ368549- } \\
\text { GQ368564 }\end{array}$ & $\begin{array}{l}\text { Nathan P. Havil } \\
\text { Adalgisa Caccone }\end{array}$ \\
\hline Artemisia tridentata & 8 & $\mathrm{n} / \mathrm{a}$ & $41279-41286$ & $\begin{array}{l}\text { AB488553- } \\
\text { AB488560 }\end{array}$ & $\begin{array}{l}\text { S. Ishizaki } \\
\text { S. Kubota } \\
\text { K. Shiojiri } \\
\text { R. Karban } \\
\text { M. Ohara }\end{array}$ \\
\hline Astroides calycularis & 13 & $\begin{array}{l}\text { Tubastrea } \\
\text { micrantha, } \\
\text { Tubastrea sp. 1, } \\
\text { Tubastrea sp. 2, } \\
\text { Tubastrea sp. } 3\end{array}$ & $41292-41304$ & $\begin{array}{l}\text { GQ292717- } \\
\text { GQ292725, } \\
\text { GQ496302- } \\
\text { GQ496305 }\end{array}$ & $\begin{array}{l}\text { P. Casado-Amezúa } \\
\text { I. Acevedo } \\
\text { R. García-Jiménez } \\
\text { A. Machordom }\end{array}$ \\
\hline Azorella selago & 8 & $\mathrm{n} / \mathrm{a}$ & $42478-42485$ & $\begin{array}{l}\text { GQ3651674- } \\
\text { GQ3651681 }\end{array}$ & $\begin{array}{l}\text { Céline Born } \\
\text { Mélodie A. McGeoch } \\
\text { Bettine Jansen van Vuuren }\end{array}$ \\
\hline $\begin{array}{l}\text { Botryllus schlosseri, } \\
\text { Botrylloides violaceus }\end{array}$ & 28 & $\begin{array}{l}\text { Botrylloides } \\
\text { fuscus, } \\
\text { Botrylloides } \\
\text { simodensis }\end{array}$ & $38070-38097$ & $\begin{array}{l}\text { GQ272527- } \\
\text { GQ272554 }\end{array}$ & $\begin{array}{l}\text { Dan G. Bock } \\
\text { Abisola A. Adebayo } \\
\text { Emmanuel E. Egbosimba } \\
\text { Melania E. Cristescu }\end{array}$ \\
\hline $\begin{array}{l}\text { Cardiocrinum } \\
\text { cordatum var. glehnii }\end{array}$ & 13 & $\mathrm{n} / \mathrm{a}$ & $41315-41327$ & $\begin{array}{l}\text { AB512096- } \\
\text { AB512108 }\end{array}$ & $\begin{array}{l}\text { M. Nishizawa } \\
\text { S. Kubota } \\
\text { M. Ohara }\end{array}$ \\
\hline $\begin{array}{l}\text { Campylopterus } \\
\text { curvipennis }\end{array}$ & 10 & $\begin{array}{l}\text { C. hemileucurus, } \\
\text { C. rufus, } \\
\text { C. largipennis, } \\
\text { C. villaviscensio, } \\
\text { Phaethornis } \\
\text { longuemareus, } \\
\text { Florisuga mellivora, } \\
\text { Lampornis } \\
\text { amethystinus, } \\
\text { Amazilia cyanocephala, } \\
\text { Archilochus colubris }\end{array}$ & $41305-41314$ & $\begin{array}{l}\text { GQ294539- } \\
\text { GQ294550 }\end{array}$ & $\begin{array}{l}\text { Clementina Gonzalez } \\
\text { Carla Gutierrez-Rodriguez } \\
\text { Juan Francisco Ornelas }\end{array}$ \\
\hline Colocasia esculenta & 19 & $\mathrm{n} / \mathrm{a}$ & $38144-38162$ & $\begin{array}{l}\text { FJ895330- } \\
\text { FJ895348 }\end{array}$ & $\begin{array}{l}\text { Wansha Li } \\
\text { Yan Zhou } \\
\text { Yongping Yang } \\
\text { Xiangyang Hu }\end{array}$ \\
\hline $\begin{array}{l}\text { Cynomys ludovicianus, } \\
\text { C. leucurus, } \\
\text { C. gunnisoni }\end{array}$ & 9 & $n / a$ & 38175-38184 & $\begin{array}{l}\text { FJ971631- } \\
\text { FJ971639, } \\
\text { FJ997263, } \\
\text { FJ980459- } \\
\text { FJ980464 }\end{array}$ & $\begin{array}{l}\text { Loren C. Sackett } \\
\text { Lianna K. Etchberger } \\
\text { Maxwell N. Mazzella } \\
\text { Douglas D. Lim } \\
\text { Andrew P. Martin }\end{array}$ \\
\hline Epinephelus coioides & 14 & $\begin{array}{l}\text { Epinephelus } \\
\text { lanceolatus, } \\
\text { Epinephelus } \\
\text { fuscoguttatus }\end{array}$ & 37966-37979 & $\begin{array}{l}\text { GQ267993- } \\
\text { GQ267993 } \\
\text { GQ381271, } \\
\text { GQ429007- } \\
\text { GQ429009 }\end{array}$ & $\begin{array}{l}\text { Le Wang } \\
\text { Zining Meng } \\
\text { Bin Fan } \\
\text { Qing Sang } \\
\text { Yayan Luo } \\
\text { Yong Zhang } \\
\text { Xiaochun Liu } \\
\text { Haoran Lin }\end{array}$ \\
\hline
\end{tabular}


Table 1 (Continued).

\begin{tabular}{|c|c|c|c|c|c|}
\hline Species & $\begin{array}{l}\text { No. of } \\
\text { primers } \\
\text { developed }\end{array}$ & $\begin{array}{l}\text { Other } \\
\text { species } \\
\text { tested }\end{array}$ & $\begin{array}{l}\text { MER } \\
\text { database } \\
\text { no. }\end{array}$ & $\begin{array}{l}\text { GenBank } \\
\text { Accession } \\
\text { no. }\end{array}$ & Authors \\
\hline Eunicella singularis & 12 & $\begin{array}{l}\text { Symbiodinium } \\
\text { temperate-A clade }\end{array}$ & $41272-41291$ & $\begin{array}{l}\text { FJ917540-FJ917550, } \\
\text { FJ919777 }\end{array}$ & $\begin{array}{l}\text { J. Cataneo } \\
\text { M. F. Ortu } \\
\text { P. Furla } \\
\text { D. Forcioli }\end{array}$ \\
\hline Gammarus pulex & 8 & $\begin{array}{l}\text { Gammarus fossarum, } \\
\text { Gammarus roeselii, } \\
\text { Dikerogammarus villosus, } \\
\text { Limnomysis benedeni }\end{array}$ & $41336-41343$ & $\begin{array}{l}\text { EH268406, } \\
\text { EH269344, } \\
\text { EH271322, } \\
\text { EH271465, } \\
\text { EH271889, } \\
\text { EH272785, } \\
\text { EH274528, } \\
\text { EH275159 }\end{array}$ & $\begin{array}{l}\text { René Gergs } \\
\text { Karl-Otto Rothhaupt } \\
\text { Jasminca } \\
\text { Behrmann-Godel }\end{array}$ \\
\hline $\begin{array}{l}\text { Homoeosoma } \\
\text { nebulella }\end{array}$ & 9 & $\mathrm{n} / \mathrm{a}$ & $38098-38106$ & $\begin{array}{l}\text { GQ150803- } \\
\text { GQ150811 }\end{array}$ & $\begin{array}{l}\text { Ling-Zhen Cao } \\
\text { Qin Ren } \\
\text { Xiang-Li Xu } \\
\text { Qing-Wen Zhang }\end{array}$ \\
\hline Hyla squirella & 11 & $\mathrm{n} / \mathrm{a}$ & $42486-42496$ & $\begin{array}{l}\text { GQ438807- } \\
\text { GQ438817 }\end{array}$ & $\begin{array}{l}\text { Tyler D. Hether } \\
\text { Eric A. Hoffman }\end{array}$ \\
\hline $\begin{array}{l}\text { Lateolabrax } \\
\text { japonicus }\end{array}$ & 11 & $\mathrm{n} / \mathrm{a}$ & $42459-42469$ & $\begin{array}{l}\text { GQ455996 } \\
\text { GQ455997 } \\
\text { GQ456002 } \\
\text { GQ456006 } \\
\text { GQ456007 } \\
\text { GQ456013 } \\
\text { GQ456018 } \\
\text { GQ456019 } \\
\text { GQ456022 } \\
\text { GQ456032 } \\
\text { GQ456037 }\end{array}$ & $\begin{array}{l}\text { Y. Zhao } \\
\text { X.S. Ji } \\
\text { H. Wang } \\
\text { Y. Q. Zeng } \\
\text { J. T. Wang }\end{array}$ \\
\hline $\begin{array}{l}\text { Mastomys } \\
\text { erythroleucus }\end{array}$ & 12 & $\mathrm{n} / \mathrm{a}$ & 38163-38174 & $\begin{array}{l}\text { GQ406216- } \\
\text { GQ406227 }\end{array}$ & $\begin{array}{l}\text { Philippe Gauthier } \\
\text { Patricia O'Brien } \\
\text { Laurent Granjon } \\
\text { Doukary Abdoullaye } \\
\text { Carine Brouat } \\
\text { Gauthier Dobigny }\end{array}$ \\
\hline Pararge aegeria & 10 & $\mathrm{n} / \mathrm{a}$ & $41344-41353$ & $\begin{array}{l}\text { FJ899644-FJ899647, } \\
\text { FJ899649-FJ899651, } \\
\text { GQ847528- } \\
\text { GQ847530 }\end{array}$ & $\begin{array}{l}\text { P. Helsen } \\
\text { S. Vandewoestijne } \\
\text { S. Van Dongen } \\
\text { E. Matthysen }\end{array}$ \\
\hline Pardosa sierra & 10 & $\mathrm{n} / \mathrm{a}$ & 37996-38005 & $\begin{array}{l}\text { EU580603- } \\
\text { EU580608, } \\
\text { FJ975139- } \\
\text { FJ975142 }\end{array}$ & $\begin{array}{l}\text { M. M. Correa-Ramirez } \\
\text { F. J. Garcia de Leon } \\
\text { M. L. Jimenez }\end{array}$ \\
\hline $\begin{array}{l}\text { Phoenicopterus } \\
\text { ruber ruber }\end{array}$ & 9 & $n / a$ & $38108-38116$ & $\begin{array}{l}\text { GQ219786- } \\
\text { GQ219790, } \\
\text { GQ379053- } \\
\text { GQ379055, } \\
\text { GQ221667 }\end{array}$ & $\begin{array}{l}\text { R. Kapil } \\
\text { G. M. Sawyer } \\
\text { L. Preston } \\
\text { R. C. Benjamin }\end{array}$ \\
\hline Silene latifolia & 8 & $\mathrm{n} / \mathrm{a}$ & $41328-41335$ & $\begin{array}{l}\text { FJ573199, } \\
\text { FJ573200, } \\
\text { FJ573202- } \\
\text { FJ573204, } \\
\text { FJ573206, } \\
\text { FJ573207, } \\
\text { FJ573209 }\end{array}$ & $\begin{array}{l}\text { Peter D. Fields } \\
\text { Stephen R. Keller } \\
\text { Pär K. Ingvarsson } \\
\text { Amy B. Pedersen } \\
\text { Douglas R. Taylor }\end{array}$ \\
\hline
\end{tabular}


Table 2 Information on the focal species, the sequencing primer pairs developed, the number of single nucleotide polymorphisms observed and any other species the loci were tested in. The next columns contain the number of allele specific primers and probes developed, and the Molecular Ecology Resources database and GenBank accession numbers, respectively. The authors responsible for each set of loci are listed in the final column

\begin{tabular}{|c|c|c|c|c|c|c|}
\hline Species & $\begin{array}{l}\text { No. of } \\
\text { primer } \\
\text { pairs }\end{array}$ & $\begin{array}{l}\text { No. of SNPs } \\
\text { in sequence }\end{array}$ & $\begin{array}{l}\text { No. of allele } \\
\text { specific } \\
\text { primers/probe }\end{array}$ & $\begin{array}{l}\text { MER } \\
\text { database } \\
\text { no. }\end{array}$ & $\begin{array}{l}\text { Genbank } \\
\text { Accession } \\
\text { no. }\end{array}$ & Authors \\
\hline $\begin{array}{l}\text { Neophocaena } \\
\text { phocaenoides }\end{array}$ & 72 & 137 & 52 & $38006-38040$ & $\begin{array}{l}\text { FI592654, } \\
\text { FI592658-FI592662, } \\
\text { FI592665, } \\
\text { FI592667, } \\
\text { FI592668, } \\
\text { FI592670, } \\
\text { FI592671, } \\
\text { F5592673, } \\
\text { FI592678, } \\
\text { FI592680-FI592688, } \\
\text { FI592690, } \\
\text { FI592691, } \\
\text { FI592693-FI592697, } \\
\text { FI592699-FI592704, } \\
\text { FI592706-FI592711, } \\
\text { FI592713, } \\
\text { FI592714, } \\
\text { F5592717 }\end{array}$ & $\begin{array}{l}\text { Shuzhen Li } \\
\text { Heyi Ji } \\
\text { Guang Yang }\end{array}$ \\
\hline
\end{tabular}

